\title{
3D Measurements in Cargo Inspection with a Gamma-Ray Linear Pushbroom Stereo System
}

\author{
Zhigang Zhu*, Li Zhao and Jiayan Lei \\ Department of Computer Science \\ The City College, The City University of New York \\ New York, NY 10031 \\ *Email: zzhu@ccny.cuny.edu
}

\begin{abstract}
In this paper we present a practical approach for $3 D$ measurements in gamma-ray (or X-ray) cargo inspection. The linear pushbroom sensor model is used for such a gamma-ray scanning system. Thanks to the constraints of the real scanning system, we model the system by using a linear pushbroom model with only one rotation angle instead of three. This greatly simplifies the calibration procedure and increases the robustness of the parameter estimation. Using only the knowledge of the dimensions of a cargo container, we automatically calibrate the sensor and find all the sensor parameters, including the image center, the focal length, the $3 D$ sensor starting location, the viewing direction, and the scanning speed. Then, a semi-automated stereo reconstruction approach is proposed to obtain $3 D$ measurements of objects inside the cargo by using two such scanning systems with different scanning angles to construct a pushbroom stereo system. Experimental results of $3 D$ measurements and visualization of a $3 D$ cargo container and the objects inside are presented. With both the interactive matching procedure and the $3 D$ visualization interface, the $3 D$ measurements could add more value to today's cargo inspection systems.
\end{abstract}

\section{Introduction}

With the ongoing development of international trade, cargo inspection becomes more and more important. Quite a few X-ray or gamma-ray cargo inspection systems have been put into practical uses (Hardin, 2002; Hardin, 2004; Hitachi, 2004). In this paper a non-intrusive gamma-ray imaging system (Orphan, et al, 2002) will be used as an example to describe our research work. This system produces gamma-ray radiographic images, and has been used for the evaluation of the contents of trucks, containers, cargo, and passenger vehicles to determine the possible presence of many types of contraband. In the past, however, cargo inspection systems have only had two- dimensional capabilities, and human operators made most of the measurements. But if we could build an accurate geometry model for the gamma-ray imaging system, which turns out to be a linear pushbroom scanning sensor, accurate three-dimensional (3D) measurements of the object inside a cargo container can be obtained when two such scanning systems with different scanning angles are used to construct a linear pushbroom stereo system. The 3D measurements add more value to today's cargo inspection techniques, as indicated in some online reports (Hardin, 2002; Hardin, 2004; Hitachi, 2004).

Pushbroom images (or mosaics, when generated from video sequences) with parallel-perspective projections are very suitable for such surveillance and/or security applications where the motion of the camera has a dominant translational direction. Examples include satellite pushbroom imaging (Gupta \& Hartley, 1997), airborne video surveillance (Zhu, et al, 2001, 2004), 3D reconstruction for image-based rendering (Chai \& Shum, 2000), road scene representations (Zheng \& Tsuji, 1992; Zhu \& Hanson, 2004), under-vehicle inspection (Dickson, et al, 2002; Koschan, et al, 2004), and 3D measurements of industrial parts by an X-ray scanning system (Gupta, et al, 1994; Noble, et al, 1995). A pushbroom image/mosaic is a parallel-perspective image, which has parallel projection in the direction of the camera's motion and perspective projection in the direction perpendicular to that motion. A pair of pushbroom stereo images/mosaics can be used for both 3D viewing and $3 \mathrm{D}$ reconstruction when they are obtained from two different oblique viewing angles. An advantageous feature of the pushbroom stereo is that depth resolution is independent of depth (Chai \& Shum, 2000; Zhu, et al 2001). This advantage can be further explained when the pushbroom stereo images are generated from a video sequence ( $\mathrm{Zhu}$, et al 2004). Since a fixed angle between the two sets of parallel viewing rays is selected for generating the stereo mosaics, for any point in the left mosaic searching for 
the match point in the right mosaic means virtually finding an original frame in which this matched pair has a fixed disparity and an adaptive baseline, depending on the depth of the point. Therefore, better depth resolution could be achieved than with perspective stereo or the recently developed multiperspective stereo with circular projection (Peleg, et al, 2001; Shum \& Szeliski, 1999; Klette, et al, 2001), given the same image resolution. We note that multiperspective stereo with circular projection that is based on wide-baseline line cameras can achieve very accurate depth resolution for far-range airborne scenes (Klette, et al, 2001). However in such a configuration, depth resolution is still proportional to the square of depth, therefore the depth accuracy varies for the cargo inspection case with large depth variations. In addition, the circular motion that is required is not the best form for scanning long cargo containers.

Using pushbroom stereo images/mosaics for $3 \mathrm{D}$ viewing and/or 3D reconstruction has been studied for satellite imaging, airborne video mosaicing, undervehicle inspection, street scene modeling, and industrial quality assurance. In this paper, issues on 3D measurements using a linear pushbroom stereo system are studied for gamma-ray cargo inspection (Orphan, et al, 2002). The closest work to ours is the $x$-ray metrology for industrial quality assurance (Noble, et al, 1995). However, to our knowledge, this paper presents the first piece of work in using linear pushbroom stereo for 3D gamma-ray or X-ray inspection of large cargo containers. This paper uses the gamma-ray scanning images provided by the Science Applications International Corporation (SAIC) (Orphan, et al, 2002). However, this does not imply an endorsement of this gamma-ray technology over others, for example, the X-ray technologies. In fact, the algorithms developed in this paper can be used for pushbroom images acquired by X-ray or other scanning approaches as well.

This paper is organized as follows. Section 2 introduces the geometry of the pushbroom scanning sensor model. In Section 3, the geometry of the pushbroom stereo system is developed. Then in Section 4, a calibration method is proposed to find the important parameters of the sensor model. In Section 5, a semi-automated stereo matching algorithm is developed. A user interface is designed to correct the matches that are initially found by a correlation-based stereo matching method. Three-dimensional (3D) reconstruction and visualization results with the outlines of a 3D cargo container and the inside objects are presented. Finally, we conclude our work and discuss a few future directions in both research and applications in Section 6.

\section{2. $\gamma$-Ray Linear Pushbroom Sensor Model}

The system diagram of the gamma-ray cargo inspection system is shown in Figure 1. A 1D detector array of $256 \mathrm{NaI}-\mathrm{PMT}$ probes counts the gamma-ray photons passing through the vehicle/cargo under inspection from a gamma-ray point source. Either the vehicle/cargo or the gamma-ray system (the source and the detector) moves in a straight line in order to obtain a 2D scanning of gamma-ray images.

The geometry of the system is shown in Figure 2. The 1D detector array geometry can be modeled by the well-known perspective projection camera $X_{c} Y_{c} Z_{c}$ with the optical center at the location of gamma-ray source, and the 1D detector array in the vertical direction $v$ and at a distance $f$ along the optical axis $Z_{c}$ (i.e., $f$ is the focal length in pixels). Note that in Figure 2 the image is drawn between the objects and the optical center for easy illustration. The scanning begins when the optical center of the sensor is at location $T=\left(T_{x}, T_{y}, T_{z}\right)$ in the world coordinate system $o-x y z$. The angle between the optical axis $\left(T Z_{c}\right)$ of the sensor and the $o z$ axis of the world coordinate is $\theta$. We assume there are no tilt and roll angles between the two coordinate systems. The sensor moves at a constant speed $S$ (feet per scan) in the direction of the $\mathrm{x}$-axis, so the velocity vector represented in the camera coordinate system $X_{c} Y_{c} Z_{c}$ is $V=\left(V_{x}, V_{y}, V_{z}\right)=(S \cos \theta, 0, S \sin \theta)$. The center of the linear image in the $v$ direction is defined by a vertical offset $\mathrm{p}_{\mathrm{v}}$. Putting all of these into the linear pushbroom projection equation formulated by Gupta \& Hartley, 1997, we have the relationship between a 3D point $(x, y, z)$ in the world coordinate system and its image point $(u, v)$ as
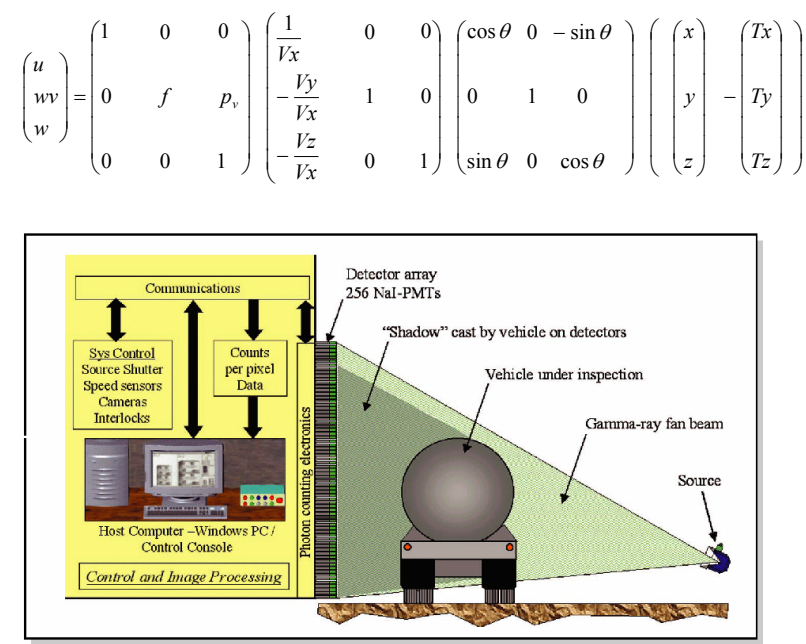

Figure 1. A gamma-ray cargo inspection system (Courtesy SAIC, San Diego, CA, USA) 


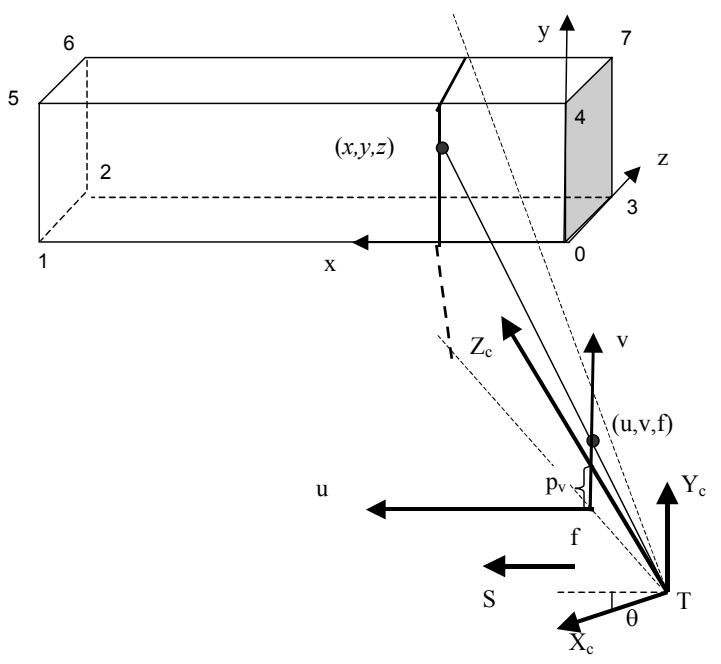

Figure 2. Linear pushbroom sensor model

This linear pushbroom equation leads to the following two simpler equations

$$
u=\frac{x-T x-(z-T z) \tan \theta}{S}
$$

and

$$
v=f \cos \theta \frac{y-T y}{z-T z}+p_{v}
$$

Note that the pushbroom scanning system has parallel projection in the $u$ direction (Eq. (1)), but has perspective geometry in the $v$ direction (Eq. (2)). Figure 3 shows three real gamma-ray images, with three different scanning angles - zero, ten and twenty degrees, respectively. Each image has a size of $621 \times 256$ pixels, i.e., 621 scans of the 256-pixel linear images.

\section{3. $\gamma$-Ray Linear Pushbroom Stereo}

A dual-scanning system is a linear pushbroom stereovision system. It can be constructed with two approaches: two linear pushbroom scanning sensors with different scanning angles, or a single scanning sensor to scan the same cargo twice with two different scanning directions. For a 3D point $(\mathrm{x}, \mathrm{y}, \mathrm{z})$, its image correspondences in the stereo pair can be represented by

$$
\begin{aligned}
& u_{k}=\frac{x-T_{x k}-\left(z-T_{z k}\right) \tan \theta_{k}}{S_{k}} \quad(\mathrm{k}=1,2) \\
& v_{k}=f_{k} \cos \theta_{k} \frac{y-T_{y k}}{z-T_{z k}}+p_{v k}
\end{aligned}
$$

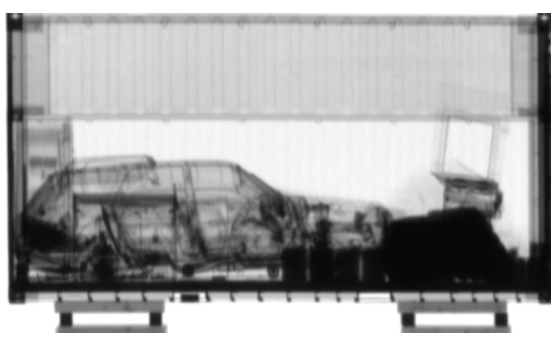

(a)

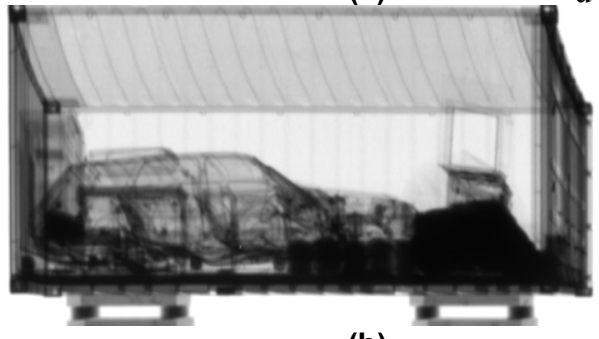

$\mathrm{u}$

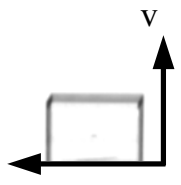

(b)

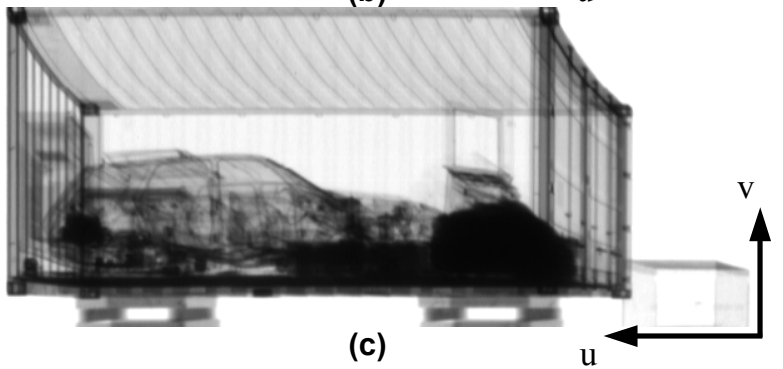

(c)

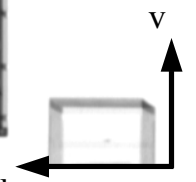

Figure 3. Real gamma-ray images with three different scanning angles (a) zero (b) ten and (c) twenty degrees (Courtesy SAIC, San Diego, CA, USA). Each image has a size of $621 \times 256$ pixels, i.e., 621 scans of the 256-pixel linear images. The stereo visual displacements, particularly the back surface of the cargo container, are obvious by comparing the three mages.

Therefore the depth of the point can be recovered as

$$
z=\frac{d-d_{0}}{\tan \theta_{1}-\tan \theta_{2}}
$$

where

$$
d=S_{2} u_{2}-S_{1} u_{1}
$$

is the visual displacement (measured in feet) of the point $(x, y, z)$ measured in the pair of stereo images, and $d_{0}=\left(T_{x 2}-T_{z 2} \tan \theta_{2}\right)-\left(T_{x 1}-T_{z 1} \tan \theta_{1}\right)$ is the fixed offset between two images. In Figure 3, the visual displacements, particularly the back surface of the cargo container, are obvious by comparing these three images. Note that Eq. (4) is acquired by only using the $u$ coordinates of the stereo images (Eq. (5)), and the depth of any point is proportional to its visual 
displacement in the stereo pair. Thus, the depth resolution is independent of depth.

After the depth is obtained via the pushbroom stereo, the $x$ and $y$ coordinates of the point can be calculated from one of the two images, for example

$$
\begin{aligned}
& x=u_{1} S_{1}+z \tan \theta_{1}+T_{x 1}-T_{z 1} \tan \theta_{1} \\
& y=\frac{\left(v_{1}-p_{v 1}\right)\left(z-T_{z 1}\right)}{f_{1} \cos \theta_{1}}+T_{y 1}
\end{aligned}
$$

\section{Sensor Calibration}

In order to use two scanning systems to calculate 3D information, we need to calibrate each scanning the system first. For each scanning setting, the following parameters are required for 3D estimation: the focal length $f$, the image center $p_{v}$, the scanning angle $\theta$, the scanning speech $S$, and the initial sensor location $\left(T_{x}, T_{y}, T_{z}\right)$. In order to fulfill this task, we need to know a set of 3D points $\left\{\left(x_{i}, y_{i}, z_{i}\right)\right\}$ and their corresponding image points $\left\{\left(u_{i}, v_{i}\right)\right\}, i=1,2, \ldots, N$. Our calibration method only needs to know the dimension of the container, which is

$$
\text { length }(\mathrm{x}) * \text { height }(\mathrm{y}) * \operatorname{depth}(\mathrm{z})=20 * 8 * 8\left(\mathrm{ft}^{3}\right)
$$

Then we locate the 8 vertices of the rectangular container (refer to Figure 2) in each gamma-ray image by manually picking up the 8 corresponding image points.

An interesting property of the linear pushbroom sensor is that Eq. (1) and Eq. (2) can work independently. Therefore, in calibrating the sensor, we first obtain the "parallel projection parameters" in Eq. (1) and then the "perspective projection parameters" in Eq. (2). Eq. (1) can be turned into a linear equation with three unknowns, i.e., $S, \tan \theta$ and $T_{x}-T_{z} \tan \theta$.

$$
u_{i} S+z_{i} \tan \theta+\left(T_{x}-T_{z} \tan \theta\right)=x_{i}
$$

Given more than three pairs of points $(i=1,2, \ldots, N$ where $N \geq 3$ )., we can solve the linear system to find the three unknowns by using the least square method. Similarly, Eq. (2) leads to a linear equation with five unknowns, i.e. $f, f T_{y}, p_{v}, p_{v} T_{z}$ and $T_{z}$ :

$$
\left(y_{i} \cos \theta\right) f-\cos \theta\left(f T_{y}\right)+z_{i} p_{v}-\left(p_{v} T_{z}\right)+v_{i} T_{z}=v_{i} z_{i}(8)
$$

With the known $\theta$ and given more than five pairs of points $(i=1,2, \ldots, N$ where $N \geq 5)$, we can solve the linear equation system. Note that from Eq. (7) we can only find the values of the speed $S$ and the angle $\theta$ and a combined parameter $T_{x}-T_{z} \tan \theta$. Nevertheless, this is sufficient for obtaining the depths of points using Eq. (4). Table 1 shows the results of the "parallel parameters" for all the three settings corresponding to images $\mathrm{a}, \mathrm{b}$, and $\mathrm{c}$ in Figure 3. All the rest of the parameters, including Tx, can be obtained after solving Eq. (8), in order to calculate the $x$ and $y$ coordinates of $3 \mathrm{D}$ points by using Eq. (6). Table 2 shows the "perspective parameters" and the $T_{x}$ values for all the three settings.

Table 3 shows the 3D measurements using the image point pairs used for calibration between two views, the ten-degree and the twenty-degree images. The purpose is to show how accurate the pushbroom stereo model and the calibration results are. The numbers of the points listed in Table 3 are labeled in Figure 2 for comparison. For the container with a dimension of $20 \times 8 \times 8 \mathrm{ft}^{3}$, the average errors in depth $\mathrm{z}$, length $\mathrm{x}$ and height $\mathrm{y}$ are $0.064 \mathrm{ft}, 0.033 \mathrm{ft}$ and $0.178 \mathrm{ft}$ respectively, indicating that the pushbroom modeling and calibration is accurate enough for 3D measurements. Note that the accuracy of the estimation only reflects the errors in sensor modeling and calibration. No image localization errors are included. The depth error $\delta z$ introduced by image localization error $\delta u$ can be estimated as the first derivative of $z$ with respect to $u$ using Eqs. (3) and (4), that is

$$
\delta z=\frac{S}{\tan \theta_{1}-\tan \theta_{2}} \delta d
$$

In Eq. (9) we assume that two scans share the same speed (i.e. $S_{1}=s_{2} \triangleq S$ ), which are almost true for our example in Figure 3. In this example, one-pixel image localization error introduces an error of $0.254 \mathrm{ft}$ in depth estimation, using the parameters in Table 1.

We have three notes about the calibrations results.

(1) The parallel parameters estimation: the estimated speeds for scanning the three images are almost the same ( $\mathrm{S}=0.0456-0.0458)$, and the angles obtained are very close to the parameters provided by the vendor (SAIC), i.e., 0, 10 and 20 degrees.

(2) The perspective parameters estimation: the three sets of parameters, including the focal lengths, the image centers, and the camera initial locations are consistent with each other.

(3) The parallel parameters are more accurate than the perspective ones due to fewer parameters in calibration and no inter-dependency among unknowns in the former, whereas three of the five unknowns in Eq. (8) are not independent, thus creating larger errors in the estimations of the $y$ coordinates than the $x$ 
coordinates (Table 3). In solving Eq. (8) using SVD, we found that the one of the four singular values of the matrix $\mathbf{A}^{\mathbf{T}} \mathbf{A}$ is almost zero, where $\mathbf{A}$ is the coefficient matrix of the linear system of Eq. (8). Therefore, the pseudo inverse of matrix $\mathbf{A}^{\mathbf{T}} \mathbf{A}$ was used.

\section{Table 1. Parallel projection parameters}

\begin{tabular}{|l|l|l|l|l|}
\hline Img & $S($ ft/pixel) & $\tan \theta$ & $\theta$ (degrees) & $T_{x}-T_{z} \tan \theta$ \\
\hline $\mathrm{A}$ & 0.04584 & 0.00143 & 0.0821 & -7.398 \\
\hline $\mathrm{b}$ & 0.04566 & 0.16552 & 9.3986 & -7.283 \\
\hline $\mathrm{c}$ & 0.04561 & 0.34493 & 19.031 & -7.309 \\
\hline
\end{tabular}

Table 2. Perspective projection parameters

\begin{tabular}{|l|l|l|l|l|l|l|}
\hline Img & $F(p x l)$ & $T_{y}(f t)$ & $p_{v}(p x l)$ & $p_{v} T_{z}$ & $T_{z}(f t)$ & $T_{x}(f t)$ \\
\hline a & 427.78 & -0.41558 & 21.148 & -177.78 & -14.815 & -7.419 \\
\hline $\mathrm{b}$ & 441.24 & -0.42881 & 17.787 & -191.78 & -15.141 & -9.789 \\
\hline c & 456.18 & -0.41037 & 19.250 & -198.03 & -15.000 & -12.48 \\
\hline
\end{tabular}

Table 3. 3D measurements of the test points

\begin{tabular}{|l|l|l|l|l|l|l|}
\hline No & $X$ & $Y$ & $Z$ & $d X$ & $d Y$ & $d Z$ \\
\hline 0 & -0.033 & -0.179 & -0.063 & -0.033 & -0.179 & -0.063 \\
\hline 1 & 20.033 & -0.177 & 0.063 & 0.033 & -0.177 & 0.063 \\
\hline 2 & 19.967 & -0.152 & 7.936 & -0.033 & -0.152 & 0.064 \\
\hline 3 & 0.033 & -0.204 & 8.064 & 0.033 & -0.204 & 0.064 \\
\hline 4 & -0.033 & 7.787 & -0.063 & -0.033 & -0.213 & -0.063 \\
\hline 5 & 20.033 & 7.856 & 0.063 & 0.033 & -0.144 & 0.063 \\
\hline 6 & 19.967 & 7.799 & 7.936 & -0.033 & -0.201 & 0.064 \\
\hline 7 & 0.033 & 7.844 & 8.064 & 0.033 & -0.156 & 0.064 \\
\hline
\end{tabular}

\section{3D Measurements and Visualization}

Fully automated 3D measurements of objects from gamma-ray radiographic images are difficult since the objects in the images are "transparent". Some work has been reported in literature (e.g. Mayntz, et al, 2000) in using optical flow on X-ray fluoroscopy images for restoration of motion blur, but the motion parallax in their case is small. However in our case of widely separated parallel viewing for $3 \mathrm{D}$ reconstruction, two different views will give very different object adjacencies and occlusions. This is an important issue and will be our future work. In our current work, we have tested an interactive approach for stereo matching and visualization. In the following two sub-sections, we will discuss these two aspects of 3D measurements for cargo inspection. Experimental results will be provided.

\subsection{Stereo matching}

Our semi-automated stereo matching approach includes three steps: interactive point selection, automated matching, and interactive matching correction. Instead of generating a dense "depth" map from a pair of gamma-ray images, we have designed an interactive user interface for selecting and measuring objects of interest. For the automated stereo matching step, we use sum of square difference (SSD) criterion on normalized images.

Figure 4 shows the process of semi-automated stereo matching for the pair of the ten- and twentydegree images. After a point in the first image is picked up by the user (marked by a red star in the first image of Figure 4), its match in the second image is automatically searched along the epipolar line of the pushbroom stereo, derived from Eq. (3). The search range is pre-determined from Eq. (4) by using the knowledge that all the objects are within the cargo container. The size of the correlation window can be determined by the user interactively. We have tried different window sizes $(3 \times 3,9 \times 9,11 \times 11$, etc. $)$ and found that $11 \times 11$ was the best for this example. The automated matches are marked by blue stars in the second image of Figure 4.

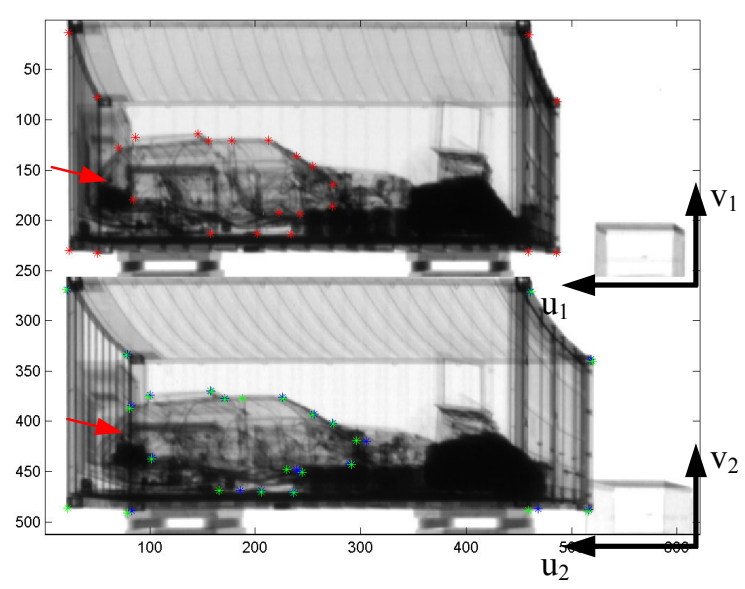

Figure 4. Pushbroom stereo matching: points selected in the left image (marked by red stars) are first searched for matches in the right image by a computer program (marked by blue stars), then the matches are corrected (if necessary) by a human operator for more accurate measurements (marked by green stars). Most of the automated matches are correct.

After the program finds the automated matching points, the user could correct the match if necessary (marked by green stars in the second image of Figure 4). In Figure 4, most of the automated matches are "considered" to be correct where the green marks completely overlap the blue marks. The points that are considered incorrect are those whose matches could be identified by human eyes but whose appearances are quite different between two images for automated matching. On the other hand, a few point matches that are considered to be "correct" might be incorrect; but 
we have no way to correct them due to the large differences between two views (e.g., the point pair pointed by arrows). In Figure 4, all eight vertices of the cargo container are selected for stereo matching as well as a few points around the boundary of a vehicle inside the cargo container. Note that the four of the eight points on the top of the container we select here are slightly different from the ones for calibration due to the requirements of an $11 \times 11$ window centered at each point.

\subsection{D visualization}

Together with the stereo matching interface, the reconstructed 3D structures are rendered as wire frames in 3D. For each set of points that are selected for stereo matching, a connected 3D line-frame representation is generated. Figure 5 shows several views of the $3 \mathrm{D}$ frame representation of the point set obtained in Figure 4: a 3D view, front view, top view, and side view. The black rectangular frame is the reconstruction of the cargo container using the calibration image data for the ten-and twenty-degrees images. The red line frame is generated from the 3D measurements by the automated stereo match algorithm. It is clearly shown that the automated stereo matches provide very good $3 \mathrm{D}$ measurements for the cargo container and the objects inside. Note that the selected points on the top of the container for the automated matches are slightly off the top so that the automated matching system can find $11 \times 11$ windows in the right images. This brings in offsets between the automated 3D estimations and their calibration references.

With a 3D visualization, 3D measurements, for example, of sizes and shapes are made simple by using the most convenient views. Object measurements and identification will be our future work.

\section{Conclusions and Discussions}

In this paper we present a practical approach for $3 \mathrm{D}$ measurements in gamma-ray (or X-ray) cargo inspection. The linear pushbroom sensor model is used for the gamma-ray scanning system. Thanks to the constraints of the real scanning system, we model the system by using a linear pushbroom model with only one rotation angle instead of three. This greatly simplifies the calibration procedure and increases the robustness of the parameter estimation. Using only the knowledge of the dimensions of the cargo container, we can automatically calibrate the sensor and find all the sensor parameters, including the image center, the focal length, the $3 \mathrm{D}$ sensor starting location, the viewing direction, and the scanning speed. The sensor modeling and calibration is accurate enough for $3 \mathrm{D}$ measurements.

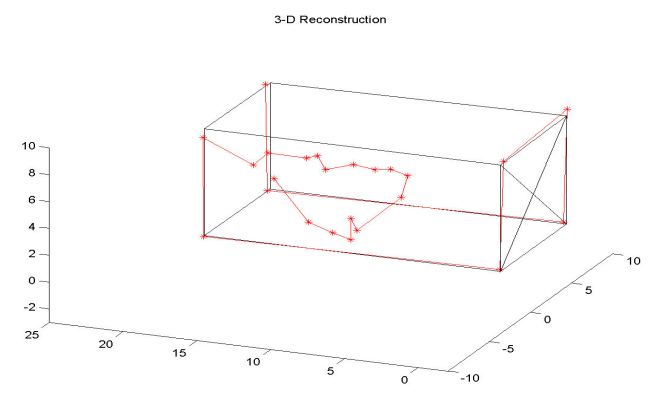

(a) 3D view

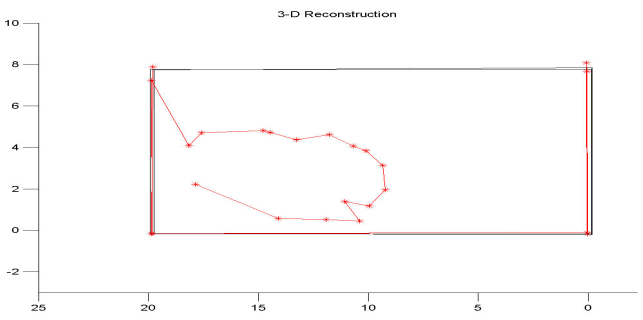

(b) Front view

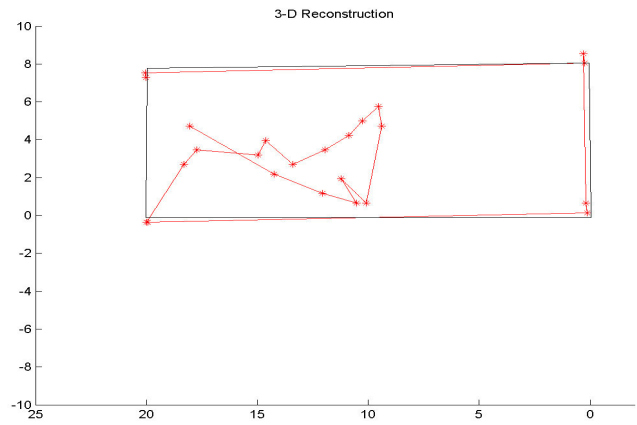

(c) Top view

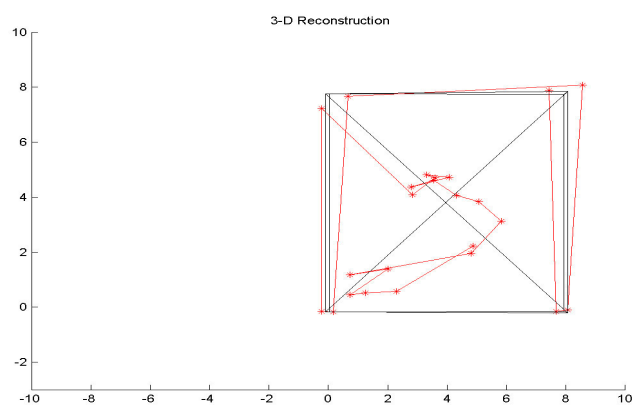

(d) Side view

Figure 5. 3D measurements and visualization of objects inside the cargo container. The black rectangular frames show the cargo container constructed from the test data in Table 3 . The red lines (with stars) show the 3D estimates from automated stereo matches, for the cargo container and an object inside. 
Then, a semi-automated stereo reconstruction approach is proposed to obtain 3D measurements of objects inside the cargo. With both the interactive matching procedure and the $3 \mathrm{D}$ visualization interface, the $3 \mathrm{D}$ measurements for cargo inspection could put into practical use.

We have made the first attempt to use pushbroom stereo for 3D gamma-ray/x-ray cargo inspection. We want to pursue this research in two directions. First, we are actively seeking collaboration with cargo inspection vendors (including SAIC) to implement more tests on real data in real deployments. By doing this we will obtain much important information that was not available when doing the current experiments, e.g. the real parameters of the sensor setting, the ground truth data of the objects under inspection. Second, we will continue our study on gamma-ray stereo matching algorithms. Most of the algorithms in literature work well only for normal visible images. However, little work exists in performing stereo matching on gamma-ray or X-ray images. The knowledge of physics and optics in generating the radiographic images could be very helpful in advancing this direction of research.

\section{Acknowledgements}

This work is partially supported by the Air Force Research Laboratory (AFRL) under the RASER Program (Award No. FA8650-05-1-1853) and an Human Effectiveness Program (Award No. F3361503-1-63-83), by Army Research Office (ARO) under Award No. W911NF-05-1-0011, and by the CUNY Graduate Research Technology Initiative (GRTI) program. We thank Dr. Rex Richardson and Dr. Victor J. Orphan at Science Applications International Corporation (SAIC) for providing us gamma-ray images and the dimension data of the cargo container. Thanks are also given to the reviewers for their comments on depth resolution of multi-perspective stereo and automated stereo matching on $\mathrm{x}$ ray/gamma-ray images, and to Mr. Robert Hill at the City College of New York for proofreading the manuscript.

\section{References}

Chai, J. and $\mathrm{H}-\mathrm{Y}$. Shum, 2000. Parallel projections for stereo reconstruction. In Proc. CVPR'00: II 493-500.

Dickson, P., J. Li, Z. Zhu, A. Hanson,, E. Riseman, H. Sabrin, H. Schultz and G. Whitten, 2002. Mosaic generation for under-vehicle inspection. IEEE Workshop on Applications of Computer Vision, Orlando, Florida, Dec 3-4, 2002
Gupta, R. and R. Hartley, 1997. Linear pushbroom cameras, IEEE Trans PAMI, 19(9), Sep. 1997: 963-975

Gupta, R., A. Noble, R. Hartley, J. Mundy, A. Schmitz, 1995. Camera calibration for 2.5-D X-ray metrology. In Proc. ICIP'95, Vol. 3, Oct 23 - 26, 1995 Washington D.C.

Hardin, W., 2002. Cargo Inspection: Imaging Solutions Wait for Government's Call, Machine Vision Online, Dec 2002.

Hardin, W., 2004. US Seaports: Finding the Needle in Hundreds of Haystacks, Machine Vision Online, June 2004.

Hitachi, 2004. Cargo container X-ray inspection systems, Hitachi Review, 53(2) June 2004: 97-102. http://www.hitachi.com/rev/field/industriasystems/2006638 $\underline{12876 . h t m l}$

Klette, R., G. Gimel'farb, R. Reulke, 2001. Wide-angle image acquisition, analysis and visualization. Proc. 14th Internat. Conf. "Vision Interface" (VI'2001), Ottawa, Canada, June 2001, 114-125.

Koschan,A., D. Page, J.-C. Ng, M. Abidi, D. Gorsich, and G. Gerhart, 2004. SAFER under vehicle inspection through video mosaic building," International Journal of Industrial Robot, September 2004, 31(5): 435-442.

Mayntz, C., T. Aach, D. Kunz and J-M. Frahm, 2000. Motion blur in fluoroscopy: effects, identification, and restoration, SPIE's Medical Imaging 2000, San Diego, CA

Noble, A., R. Hartley, J. Mundy and J. Farley. X-Ray Metrology for Quality Assurance, In Proc IEEE ICRA'94, vol 2, pp 1113-1119

Peleg S, M, Ben-Ezra and Y. Pritch, 2001. Omnistereo: panoramic stereo imaging, IEEE Trans. PAMI, 23(3): 279290.

Shum, H.-Y. and R. Szeliski, 1999. Stereo reconstruction from multiperspective panoramas. In Proc. ICCV'99: 14-21

Orphan, V. J., R. Richardson and D. W. Bowlin, 2002. VACIS $^{\mathrm{TM}}$ - a safe, reliable and cost- effective cargo inspection technology, Port Technology International, p. 6165. www.porttechnology.org/journals/ed16/section02.shtml

Zheng, J. Y., and S. Tsuji, 1992. Panoramic representation for route recognition by a mobile robot. International Journal of Computer Vision, 9(1), 1992: 55-76

Zhu, Z. and A. R. Hanson, 2004. LAMP: 3D Layered, Adaptive-resolution and Multi-perspective Panorama - a New Scene Representation, Computer Vision and Image Understanding, 96(3), Dec 2004, pp 294-326.

Zhu, Z. , E. M. Riseman and A. R. Hanson, 2001. Parallelperspective stereo mosaics. In Proc. ICCV'01, vol I: 345352.

Zhu, Z., E. M. Riseman, A. R. Hanson, 2004. Generalized Parallel-Perspective Stereo Mosaics from Airborne Videos, IEEE Trans PAMI, 26(2), Feb 2004, pp 226-237 